\title{
Holographic QCD: Status and perspectives for the future
}

\section{Shigeki Sugimoto ${ }^{* \dagger}$}

Kavli IPMU, University of Tokyo (WPI)

E-mail: shigeki.sugimotodipmu.jp

We give a brief overview of holographic QCD. Applying the idea of gauge/string duality to QCD, we obtain a low energy effective theory of hadrons based on string theory. Mesons and baryons are obtained as open strings and D-branes, respectively, and a lot of properties of hadrons can be extracted using this description.

Xth Quark Confinement and the Hadron Spectrum

8 - 12 October 2012

TUM Campus Garching, Munich, Germany

\footnotetext{
* Speaker.

${ }^{\dagger}$ Main part of this article is based on the works done in collaboration with T. Sakai, H. Hata, S. Yamato, K. Hashimoto and T. Imoto. 102, 3,5
} 


\section{Claim}

The main claim of this article is that string theory can be a theory of hadrons. For this, we do not mean that QCD has some missing ingredients to describe hadrons. Our claim is that string theory in a certain curved background can be physically equivalent to QCD at least at low energies. At first sight, the string theory description looks completely different from $\mathrm{QCD}$, and one may wonder why they can be dual to each other. The fundamental degrees of freedom in string theory are not elementary particles but strings. QCD is a gauge theory without gravity, but string theory contains gravitons as massless modes. Furthermore, string theory is defined in a ten dimensional curved background, while QCD is defined in a flat four dimensional space-time. As we will explain below, this claim is based on gauge/string duality, which has been studied extensively since the discovery of the AdS/CFT correspondence. [6] ${ }^{1}$ One of the most surprising features in gauge/string duality is that two theories defined in different space-time dimensions, (gauge theory in four (or lower) dimensions and string theory in ten dimensions), are claimed to be dual to each other. For this reason, this duality is often called a "holographic dual", and the string theory description dual to QCD is called "holographic QCD", which is the title of this article.

In holographic QCD, hadrons are described without using quarks and gluons. As explained below, mesons and baryons correspond to open strings and D-branes wrapped on a non-trivial cycle in the background, respectively. With this interpretation, we are able to analyze low energy properties of hadrons. The main purpose of this article is to report how well hadrons can be described in this approach and discuss future directions.

The article is organized is as follows. In section 2 we give a tiny review of D-branes and gauge/string duality. If you are familiar with these terminologies you can skip this section. Our model is explained in section 3 . If you just want to learn what we can obtain using holographic QCD, you can go directly to section 4 where we summarize some of the typical results. Section 5 and section 6 are the summary of the status and perspectives for the future, respectively.

\section{Gauge/string duality}

The gauge/string duality is a duality between a four dimensional gauge theory ${ }^{2}$ and string theory in a certain ten dimensional curved background. A typical example of the gauge/string duality is the duality between four dimensional $\mathscr{N}=4$ super Yang-Mills theory and type IIB superstring theory in $\mathrm{AdS}_{5} \times S^{5}$ background. Since $\mathscr{N}=4$ super Yang-Mills theory is a conformal field theory (CFT), and the background in the holographic dual description contains AdS factor, this type of duality is often called "AdS/CFT correspondence". Even for this most well-studied example, the direct proof of the equivalence between these two descriptions is still an open problem. However, since there are a large number of evidences supporting this duality, it is widely believed that they are physically equivalent. Our aim is to apply this idea to more realistic gauge theory (QCD) and see what we can learn from string theory.

To explain the basic idea of the duality, we introduce an object called D-brane in string theory. A D $p$-brane is defined as a $(p+1)$ dimensional object on which end points of open strings can be

\footnotetext{
${ }^{1}$ See, e.g., 7 for a review.

${ }^{2}$ There are examples in other dimensions, but here we only consider four dimensional gauge theories.
} 
attached. It can be shown that gauge particles living on the $\mathrm{D} p$-brane are obtained as the massless modes of the open strings attached on it. Therefore, if we consider a flat $\mathrm{D} p$-brane, a $(p+1)$ dimensional gauge theory is realized on it. For the cases with $N_{c} \mathrm{D} p$-branes located at the same place, the gauge group is enhanced to $U\left(N_{c}\right)$.

The basic idea of gauge/string duality is as follows. Consider a system with D-branes. As we have just explained, the low energy effective theory of open strings attached on the D-branes is a gauge theory. On the other hand, since D-branes couple with gravity, the space-time around them will be curved following the general principle of general relativity. In some parameter region (large $N_{c}$ and large $\lambda$ in our case discussed in the following sections), it can be shown that the D-branes are better described as the corresponding curved background. Therefore, there are two descriptions of the D-branes. One of them is a gauge theory and the other one is string theory in a curved background. Since they are describing the same D-brane system, these two descriptions are expected to be equivalent.

Note that in this naive explanation, we didn't use supersymmetry nor conformal symmetry. Therefore, it is natural to expect that such duality also holds for non-supersymmetric and nonconformal gauge theory like QCD. In general, it is more difficult to analyze a strongly coupled gauge theory with less symmetry, and hence, collecting non-trivial evidence of the gauge/string duality for such cases will be much more challenging. Fortunately, for QCD, we can skip all the complicated calculations in the gauge theory side and directly compare the results in the string theory side with the experimental data to check whether this duality works or not. Once we accept this duality, it will provide powerful tools, which are completely different from the traditional ones based on QCD, to analyze properties of hadrons.

\section{Holographic QCD}

A holographic description of Yang-Mills theory (without quarks) was proposed by Witten in [8]. He proposed to consider $N_{c}$ D4-branes extended along $x^{0 \sim 4}$ directions, where the $x^{4}$ direction is compactified to $S^{1}$ with an anti-periodic boundary condition for all the fermions in the system. The supersymmetry is completely broken by this boundary condition and it is possible to show that the low energy effective theory of the open strings attached on the D4-branes is $U\left(N_{c}\right)$ YangMills theory. To add quarks to this system, we add $N_{f}$ D8-D8 pairs extended along $x^{0 \sim 3}$ and $x^{5 \sim 9}$ directions. [1] The D8-branes and $\overline{\mathrm{D} 8}$-branes are placed at the antipodal points in the $S^{1}$ parameterized by $x^{4}$. (See the left side of Figure 1) Then, we can show that the massless degrees of freedom obtained from the open strings stretched between D4-branes and D8( or $\overline{\mathrm{D} 8}$ )-branes are $N_{f}$ massless fermions in fundamental representation of the $U\left(N_{c}\right)$ gauge group. As a result, the system flows to $U\left(N_{c}\right)$ QCD with $N_{f}$ massless quarks at low energy. ${ }^{3}$

Note that the chiral symmetry $U\left(N_{f}\right)_{L} \times U\left(N_{f}\right)_{R}$ is manifestly realized as the gauge symmetry of the D8- $\overline{\mathrm{D} 8}$ system. ${ }^{4}$ We take a limit to decouple gravity as well as the other particles that can

\footnotetext{
${ }^{3}$ We do not care much about the difference between $S U\left(N_{c}\right)$ and $U\left(N_{c}\right)$, because the $U(1)$ part of $U\left(N_{c}\right)$ will become weak at low energies and also because the contribution of the $U(1)$ part is subleading in the $1 / N_{c}$ expansion.

${ }^{4}$ The axial $U(1)$ part is anomalous. Although we will not discuss it in this article, it is possible to estimate the effect of this anomaly. See 1 for the details.
} 
move away from the D4-branes. In this limit, the gauge fields on the D8(and $\overline{\mathrm{D} 8}$ )-branes decouple from the QCD sector and the $U\left(N_{f}\right)_{L} \times U\left(N_{f}\right)_{R}$ symmetry becomes a global symmetry.

There are two parameters in the system. One of them is the radius of the $S^{1}$, denoted as $M_{\mathrm{KK}}^{-1}$, and the other parameter is the 't Hooft coupling $\lambda=g_{\mathrm{YM}}^{2} N_{c}$. Note that there are Kaluza-Klein (KK) modes with masses of order $M_{\mathrm{KK}}$, which cannot be interpreted as particles in QCD. Therefore, $M_{\mathrm{KK}}$ can be thought of as a cut-off scale below which the theory can be regarded as QCD. Note, however, that we expect the effect of this "cut-off" is much milder than that of lattice gauge theory, because we are not really cutting off the energy higher than $M_{\mathrm{KK}}$ and the Lorentz symmetry as well as the chiral symmetry are not explicitly broken.

To obtain a holographic description, we replace the D4-branes with the corresponding curved background found in [8]. Then, the radius of the $S^{1}$ becomes $r$ dependent, where $r$ is the radial coordinate of the $x^{5 \sim 9}$ plane, and we obtain a cigar-like geometry as depicted in the right side of Figure 1. The topology of the space-time is $\mathbf{R}^{1,3} \times \mathbf{R}^{2} \times S^{4}$, where $\mathbf{R}^{1,3}$ and $\mathbf{R}^{2}$ factors are

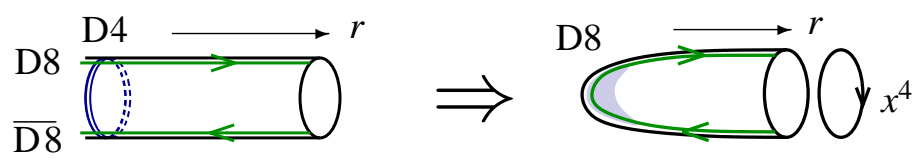

Figure 1: Replacing D4-brane with the corresponding curved background.

parameterized by $x^{0 \sim 3}$ and $\left(r, x^{4}\right)$, respectively, and $S^{4}$ corresponds to the angular directions of the $x^{5 \sim 9}$ plane. The D8-branes are treated as probe branes and we neglect their backreaction to the background. This treatment is called probe approximation and it can be justified when $N_{c} \gg N_{f}$. It is analogous to the quench approximation widely used in lattice $\mathrm{QCD}$, because we are neglecting the contributions of string world-sheet with extra boundaries attached on the D8-brane, which correspond to fermion loops in QCD. Then, the D8- $\overline{\mathrm{D} 8}$ pairs are now smoothly connected as in the right side of Figure 1. This is the setup of our holographic QCD.

As mentioned above, the $U\left(N_{f}\right)_{L} \times U\left(N_{f}\right)_{R}$ gauge symmetry of the D8-D8 system corresponds to the chiral symmetry in QCD. Since the D8-D8 pairs are connected after replacing the D4-branes with the corresponding curved background, this $U\left(N_{f}\right)_{L} \times U\left(N_{f}\right)_{R}$ is broken to $U\left(N_{f}\right)$. This phenomenon corresponds to the chiral symmetry breaking in QCD. In fact, as we will see in the next section, we can find the Nambu-Goldstone modes associated with this chiral symmetry breaking in the spectrum.

In this description, it can be shown that the string coupling is proportional to $1 / N_{c}$ and the string length is proportional to $\lambda^{-1 / 2}$. Therefore, this description can be trusted when $N_{c}$ and $\lambda$ are large enough. Since QCD is an asymptotically free theory, the latter condition $(\lambda \gg 1)$ implies that this description works better at low energy.

\section{Surprise}

Using the holographic description explained in the previous section, a lot of properties of hadrons can be extracted. Here, we just show some of the interesting results without trying to explain the details. The readers should keep in mind that the approximations we made may not be 
accurate enough. In most of our calculations, we have only estimated the leading terms in the $1 / N_{c}$ and $1 / \lambda$ expansions. Since we have not estimated the $1 / N_{c}$ and $1 / \lambda$, corrections, we cannot tell how much we can trust our results. In our quantitative results, we set $M_{\mathrm{KK}}$ to be around $1 \mathrm{GeV}$, which is not high enough to justify the decoupling of unwanted KK modes. In addition, we have neglected the effect of the quark masses. ${ }^{5}$ Therefore, we should not expect too much. If the order of magnitudes in our calculations turn out to be comparable to the experimental results, we should consider it as a success. But, please don't be too pessimistic. As we will show in the following, it turns out that the results look much better than what one would naively expect.

First of all, hadrons are nicely realized in our framework. As depicted in Figure 2 glueballs, mesons, and baryons in QCD are realized as closed strings, open strings attached on the D8-branes, and D4-branes wrapped on $S^{4}$, respectively. Since the end points of the open strings

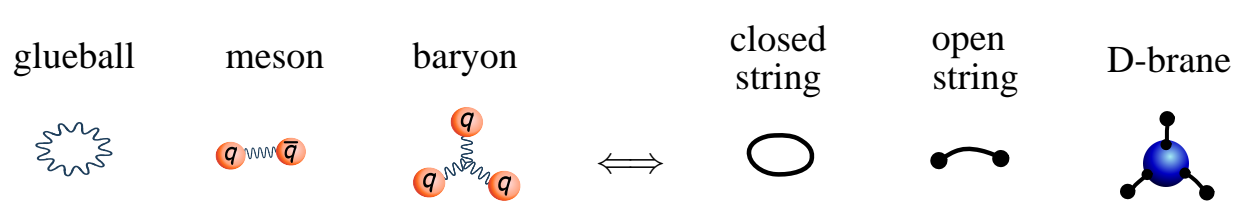

Figure 2: Hadrons in the model.

are attached on the D8-branes, each open string carries two flavor indices, which is consistent with our interpretation as a meson. Although we will not explain the details here, it is known that the D4-brane wrapped on the $S^{4}$ has to have $N_{c}$ open strings attached on it because of the RR flux in the background.[11, 12] Therefore, it is plausible to interpret this object as a bound state of $N_{c}$ quarks in QCD.

\subsection{Mesons}

The effective theory of mesons can be obtained from the effective theory of open strings attached on the D8-branes. Using the standard D8-brane effective action, and integrating over the $S^{4}$ direction, we obtain the following five dimensional $U\left(N_{f}\right)$ Yang-Mills-Chern-Simons theory:

$$
\begin{gathered}
S_{5 \mathrm{dim}} \simeq S_{\mathrm{YM}}+S_{\mathrm{CS}} \\
S_{\mathrm{YM}}=\kappa \int d^{4} x d z \operatorname{Tr}\left(\frac{1}{2} h(z) F_{\mu \nu}^{2}+k(z) F_{\mu z}^{2}\right), \quad S_{\mathrm{CS}}=\frac{N_{c}}{24 \pi^{2}} \int_{5 \operatorname{dim}} \omega_{5}(A),
\end{gathered}
$$

where $h(z)=\left(1+z^{2}\right)^{-1 / 3}$ and $k(z)=1+z^{2}$. Here $\mu, v=0 \sim 3$ correspond to the coordinates of our four dimensional world, and $-\infty<z<\infty$ is the coordinate parameterizing the D8-brane worldvolume in the right side of Figure 1 The coefficient $\kappa$ in $S_{\mathrm{YM}}$ is a constant proportional to $\lambda N_{c}$ and $\omega_{5}(A)$ in $S_{\mathrm{CS}}$ is the Chern-Simons 5-form. The five dimensional gauge field can be decomposed as

$$
A_{\mu}\left(x^{\mu}, z\right)=\sum_{n \geq 1} B_{\mu}^{(n)}\left(x^{\mu}\right) \psi_{n}(z), \quad A_{z}\left(x^{\mu}, z\right)=\sum_{n \geq 0} \varphi^{(n)}\left(x^{\mu}\right) \phi_{n}(z)
$$

using complete sets $\left\{\psi_{n}\right\}_{n \geq 1}$ and $\left\{\phi_{n}\right\}_{n \geq 0}$ of functions of $z$. The coefficient $B_{\mu}^{(n)}$ and $\varphi^{(n)}$ are four dimensional vector and scalar fields, respectively. It can be shown that the vector field $B_{\mu}^{(n)}$ becomes

\footnotetext{
${ }^{5}$ See 9010 for the analysis including quark masses.
} 
massive by absorbing $\varphi^{(n)}$, while $\varphi^{(0)}$ remain massless. Now, we interpret (the isovector part of) $B_{\mu}^{(1)}, B_{\mu}^{(2)}, B_{\mu}^{(3)}$, etc., as the $\rho$-meson, $a_{1}$-meson, $\rho^{\prime}$-meson, etc., respectively, and $\varphi^{(0)}$ as the pion. It can be easily checked that the spin, parity and charge conjugation parity are consistent in this interpretation. Another reason for interpreting $\varphi^{(0)}$ as the pion field is that the pion is considered to be the Nambu-Goldstone particle associated with the spontaneous chiral symmetry breaking and should become massless when the quarks are massless. We recover this property successfully. ${ }^{6}$

Inserting the expansions (4.2) into the five dimensional action (4.1) and integrating over $z$, we obtain a traditional four dimensional effective action of the mesons $\pi, \rho, a_{1}$, etc.:

$$
S_{5 \operatorname{dim}}(A)=S_{4 \operatorname{dim}}\left(\pi, \rho, a_{1}, \rho^{\prime}, a_{1}^{\prime}, \cdots\right) .
$$

It is not difficult to compute the masses and couplings from the effective action in 4.3). It turns out that the kinetic terms and mass terms for the mesons $B_{\mu}^{(n)}$ are diagonalized when we choose the complete set $\left\{\psi_{n}\right\}_{n \geq 1}$ to be a set of eigenfunctions satisfying the eigenequation

$$
-h(z)^{-1} \partial_{z}\left(k(z) \partial_{z} \psi_{n}(z)\right)=\lambda_{n} \psi_{n}(z)
$$

where $\lambda_{n}$ is the eigenvalue. Then, the mass of $B_{\mu}^{(n)}$ is given by $\sqrt{\lambda_{n}} M_{\mathrm{KK}}$. It can be readily shown that the eigenvalues $\lambda_{n}(n=1,2,3, \cdots)$ are all positive and non-degenerate. We choose the order such that $0<\lambda_{1}<\lambda_{2}<\cdots$. The coupling constants are obtained as the coefficients of the interaction terms in (4.3). For example, the $\rho-\pi-\pi$ coupling $g_{\rho \pi \pi}$ is obtained as

$$
g_{\rho \pi \pi}=\frac{1}{\pi} \int d z k(z)^{-1} \psi_{1}(z) .
$$

\begin{tabular}{|c|c|c|c|c|c|}
\hline & coupling & our model & \multirow{4}{*}{$\begin{array}{c}\text { experiment } \\
92.4 \mathrm{MeV} \\
(0.1 \sim 0.7) \times 10^{-3} \\
(1.1 \sim 1.7) \times 10^{-3}\end{array}$} \\
\hline & & & \multirow{2}{*}{$\begin{array}{l}f_{\pi} \\
L_{1}\end{array}$} & \multirow{2}{*}{$\begin{array}{l}{[92.4 \mathrm{MeV}]} \\
0.58 \times 10^{-3}\end{array}$} & \\
\hline & & & & & \\
\hline mass & our model & experiment & $L_{2}$ & $1.2 \times 10^{-3}$ & \\
\hline$\rho$ & [776 MeV] & $776 \mathrm{MeV}$ & $L_{3}$ & $-3.5 \times 10^{-3}$ & $-(2.4 \sim 4.6) \times 10^{-3}$ \\
\hline$a_{1}$ & $1189 \mathrm{MeV}$ & $1230 \mathrm{MeV}$ & $L_{9}$ & $8.7 \times 10^{-3}$ & $(6.2 \sim 7.6) \times 10^{-3}$ \\
\hline$\rho^{\prime}$ & $1607 \mathrm{MeV}$ & $1465 \mathrm{MeV}$ & $L_{10}$ & $-8.7 \times 10^{-3}$ & $-(4.8 \sim 6.3) \times 10^{-3}$ \\
\hline & & & $g_{\rho \pi \pi}$ & 4.8 & 6.0 \\
\hline & & & $g_{\rho}$ & $0.16 \mathrm{GeV}^{2}$ & $0.12 \mathrm{GeV}^{2}$ \\
\hline & & & $g_{a_{1} \rho \pi}$ & $4.6 \mathrm{GeV}$ & $2.8 \sim 4.2 \mathrm{GeV}$ \\
\hline
\end{tabular}

It is not difficult to evaluate these values numerically. We list some of the results in Table 1 .

Table 1: Masses and couplings calculated in our model.[1] 2] Here, $M_{\mathrm{KK}}$ and $\lambda$ are fixed by fitting the $\rho$ meson mass and the pion decay constant $f_{\pi}$.

Furthermore, it can be shown that the meson effective theory obtained in (4.3) reproduces a lot of old phenomenological models of hadrons, such as Skyrme model, vector meson dominance model, Gell-Mann - Sharp - Wagner model, hidden local symmetry model, a bottom up holographic

\footnotetext{
${ }^{6}$ It was also shown that the Gell-Mann - Oaks - Renner relation (the relation between the pion mass and quark masses) is satisfied when the quark masses are included.9
} 
model proposed in [13], etc., without making any phenomenological assumptions. The CS-term $S_{\mathrm{CS}}$ correctly reproduces the chiral anomaly in QCD and yields the WZW term.

As a typical example, let us briefly explain what we can learn for the $\omega$-meson decay.[2] Here we consider the cases with $N_{f}=2$. Then, the vector meson field $B_{\mu}^{(1)}$ obtained above is a $2 \times 2$ hermitian matrix. The trace part (isoscalar part) corresponds to the $\omega$-meson, while the other three components (isovector part) correspond to the $\rho$-meson $\left(\rho^{0}\right.$ and $\left.\rho^{ \pm}\right)$. We are interested in the decay channels $\omega \rightarrow \pi \gamma$ and $\omega \rightarrow \pi \pi \pi$. The relevant diagrams for these decay channels obtained from the effective action (4.3) are depicted in Figure 3 Note that there are no direct $\omega-\pi-\gamma$ and
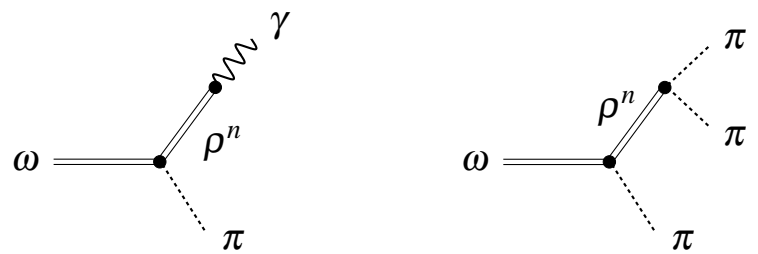

Figure 3: The relevant diagrams for $\omega \rightarrow \pi \gamma$ and $\omega \rightarrow \pi \pi \pi$. $\rho^{n}$ is the isovector part of the vector meson $B_{\mu}^{(2 n-1)}$.

$\omega-\pi-\pi-\pi$ couplings, and these decay modes are always mediated by the vector mesons $\rho^{n}$, which is the isovector part of the vector meson $B_{\mu}^{(2 n-1)}$. This structure is exactly what Gell-Mann - Sharp - Wagner proposed in [14]. Furthermore, using the completeness condition for $\left\{\psi_{n}\right\}_{n \geq 1}$, we can show a sum rule:

$$
\sum_{n=1}^{\infty} \frac{g_{\omega \rho^{n}} \pi g_{\rho^{n} \gamma}}{m_{\rho^{n}}^{2}}=g_{\rho \pi \pi}
$$

where $g_{\omega \rho^{n} \pi}, g_{\rho^{n} \gamma}$ and $g_{\rho \pi \pi}$ are the coupling constants for $\omega-\rho^{n}-\pi, \rho^{n}-\gamma$ and $\rho-\pi-\pi$ interaction, respectively, and $m_{\rho^{n}}$ is the mass of the vector meson $\rho^{n}$. Using this sum rule, the decay width of the $\omega \rightarrow \pi \gamma$ can be calculated as

$$
\Gamma(\omega \rightarrow \pi \gamma)=\frac{N_{c}^{2}}{3} \frac{\alpha}{64 \pi^{4} f_{\pi}^{2}}\left(\sum_{n=1}^{\infty} \frac{g_{\omega \rho^{n} \pi} g_{\rho^{n} \gamma}}{m_{\rho^{n}}^{2}}\right)^{2}\left|\mathbf{p}_{\pi}\right|^{3}=\frac{N_{c}^{2}}{3} \frac{\alpha}{64 \pi^{4} f_{\pi}^{2}} g_{\rho \pi \pi}^{2}\left|\mathbf{p}_{\pi}\right|^{3},
$$

which reproduces the expression given in [15].

Other mesons, including higher spin mesons, are obtained as excited string states. For example, $a_{2}(1320), b_{1}(1235), \pi(1300), a_{0}(1450)$, etc., are interpreted as the first excited open string states, and $\rho_{3}(1690), \pi_{2}(1670)$, etc., are interpreted as the second excited states. The lightest spin $J$ mesons with $J \geq 1$ are $(J-1)$-th excited open string states. See [5] for more details.

\subsection{Baryons}

As mentioned above, baryons are obtained as D4-branes wrapped on $S^{4}$. It is known that a D4brane embedded in D8-brane world-volume is equivalent to a soliton in the gauge theory realized on the D8-brane. [16] In our five dimensional gauge theory 44.1, baryons are described as a soliton carrying non-trivial instanton number

$$
\frac{1}{8 \pi^{2}} \int_{\mathbf{R}^{4}} \operatorname{Tr}(F \wedge F)=N_{B},
$$


where $\mathbf{R}^{4}$ is the four dimensional space parameterized by $\left\{\left(x^{1 \sim 3}, z\right)\right\}$ and $N_{B}$ is an integer interpreted as the baryon number. This is analogous to the description of baryons as solitons (Skyrmions) in Skyrme model [17], and more directly related to the proposal in [18], in which the Skyrmions are obtained from the instanton configurations in a five dimensional Yang-Mills theory.

Applying the techniques developed in the Skyrme model [19] to our system, we can analyze various properties of baryons. The spectrum of the baryon is obtained as in Figure 4 Our result

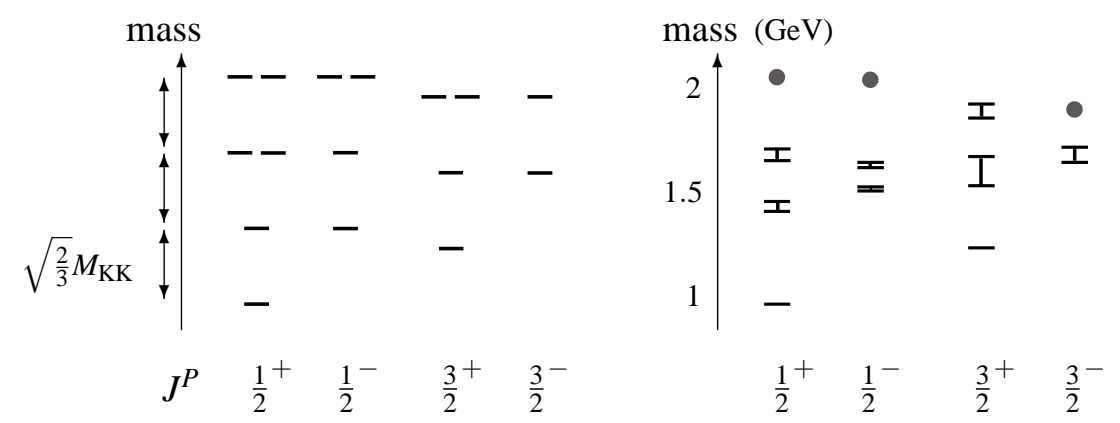

Figure 4: Left side is the baryon spectrum obtained in our model [3], while the right side is the spectrum of the baryons with $I=J$ taken from particle listings by Particle Data Group. Here $I$ and $J$ are the isospin and spin, respectively, and and $P$ is the parity.

(left side of Figure 4) seems to reproduce some of the qualitative features found in the experimental data (right side of Figure 44). For example, the lightest and second lightest baryons are the states with $J^{P}=\frac{1}{2}^{+}$and $J^{P}=\frac{3}{2}^{+}$, respectively, the second lightest baryon with $J^{P}=\frac{1}{2}^{+}\left(J^{P}=\frac{3}{2}^{+}\right)$and the lightest one with $J^{P}=\frac{1}{2}^{-}\left(J^{P}=\frac{3}{2}^{-}\right)$are almost degenerate, etc. Note that the latter feature had been a mystery in hadron physics for a long time, since it contradicts with the naive analysis based on quark model. ${ }^{7}$

By evaluating the electromagnetic current operator, we can analyze the electromagnetic properties of baryons generalizing the idea of [19] for Skyrmions. The results for the charge radii and magnetic moments for nucleons obtained in [4] are listed in Table 2$]^{8}$ It is also possible to compute the electric and magnetic form factors for baryons. A plot of the electric form factor for a proton computed in [4] is shown in Figure 5$]^{9}$ The low energy behavior seems to agree quite well with the dipole profile $\left(1+\vec{k}^{2} / \Lambda^{2}\right)^{-2}$, which is known empirically to reproduce the experimental data very well at low energies.

\section{Summary of Status}

Let us summarize here what are more or less well-understood up to now.

1. Qualitative features

\footnotetext{
${ }^{7}$ See, e.g., 20,21] for the analysis in lattice gauge theory.

${ }^{8}$ See also 22, 23, 24, 25] for related works.

${ }^{9}$ See also 26, 25] for related works.
} 


\begin{tabular}{|c||c|c|}
\hline & our model & experiment \\
\hline$\left\langle r^{2}\right\rangle_{I=0}$ & $(0.74 \mathrm{fm})^{2}$ & $(0.81 \mathrm{fm})^{2}$ \\
$\left\langle r^{2}\right\rangle_{I=1}$ & $(0.74 \mathrm{fm})^{2}$ & $(0.94 \mathrm{fm})^{2}$ \\
$\left\langle r^{2}\right\rangle_{A}$ & $(0.54 \mathrm{fm})^{2}$ & $(0.67 \mathrm{fm})^{2}$ \\
$g_{I=0}$ & 1.7 & 1.8 \\
$g_{I=1}$ & 7.0 & 9.4 \\
$g_{A}$ & 0.73 & 1.3 \\
\hline
\end{tabular}

Table 2: $\left\langle r^{2}\right\rangle_{I=0},\left\langle r^{2}\right\rangle_{I=1}$ and $\left\langle r^{2}\right\rangle_{A}$ are isoscalar, isovector and axial mean square radii, respectively. $g_{I=0}$ and $g_{I=1}$ are isoscalar and isovector g-factors. $g_{A}$ is the axial coupling.

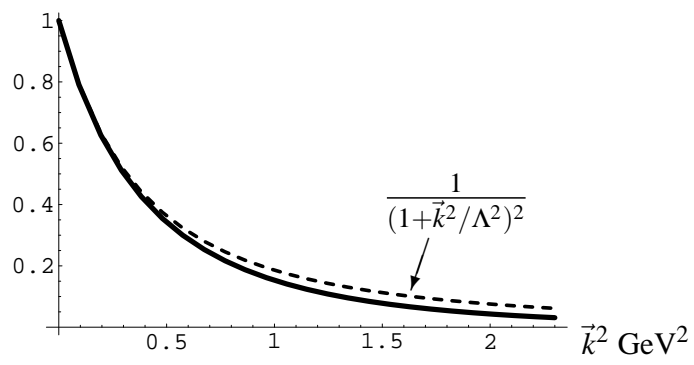

Figure 5: A plot of the electric form factor for a proton. The solid line is our result [4 and the dotted line is the dipole profile with $\Lambda^{2}=0.758 \mathrm{GeV}^{2}$.

A lot of qualitative features of QCD are reproduced in holographic QCD. For example, confinement, chiral symmetry breaking, existence of baryons and mesons, origin of baryon masses, etc. are understood quite easily without making any detailed computations, if we start with the holographic description explained in section 3 Furthermore, the holographic description provides geometric interpretations for these phenomena.

\section{Spectrum of hadrons}

As explained in section 4 glueballs, mesons and baryons correspond to closed strings, open strings and D-branes, respectively. The spectrum of the hadrons obtained from this interpretation reproduces many of the observed hadrons. Not only the pattern of quantum numbers $\left(J^{P C}\right)$, but also the masses are in reasonable agreement with the experimental data. ${ }^{10}$

\section{Structure of interaction}

Coupling constants for the interaction among mesons as well as those with baryons are calculated and compared with the experimental data. Again, we find non-trivial agreement. Furthermore, it can be analytically shown that the structure of interaction is consistent with various phenomenological models that are proposed in the past.

\footnotetext{
${ }^{10}$ See 7 27 and references therein for the analysis of the glueball spectrum obtained from the fluctuations of supergravity fields.
} 


\section{Perspectives}

There are a lot of things we should explore. Here, I only list a few of them.

\section{1. $1 / \lambda$ and $1 / N_{c}$ corrections}

As mentioned in section 4 in most of our results, we have only evaluated the leading terms in the $1 / \lambda$ and $1 / N_{c}$ expansions. It is not clear how much we can trust this approximation for the case of realistic QCD with $N_{c}=3$ and hence, it is important to evaluate the corrections. The $1 / \lambda$ and $1 / N_{c}$ corrections in QCD correspond to $\alpha^{\prime}$ and loop corrections in string theory, respectively. Therefore, in principle, if the $\alpha^{\prime}$ and loop corrections in string theory are calculated, we are able to estimate the corrections to the results shown in section 4

\section{Connection to perturbative QCD}

As sketched in section 3, our holographic QCD is supposed to be dual to a gauge theory that flows to four dimensional QCD at low energies. However, since it contains KK artifacts of masses of order $M_{\mathrm{KK}}$, it will deviate from QCD at high energies. In order to access to higher energy and connect to perturbative QCD, we should make $M_{\mathrm{KK}}$ large, while keeping the dynamical scale $\Lambda_{\mathrm{QCD}}$ fixed. Because of the asymptotic freedom of QCD, we have to make $\lambda$ small when $M_{\mathrm{KK}}$ is large. Then, the $1 / \lambda$ expansion will eventually break down and we have to deal with full stringy calculation to know the $\lambda$ dependence for small $\lambda$.

\section{Phase structure}

Needless to say, it is important to understand the phase structure of QCD. Since it is not difficult to introduce finite temperature, chemical potentials, electric/magnetic fields, etc., in holographic QCD, we expect that holographic QCD may provide useful techniques to analyze the phase structure including such external parameters. In fact, there are many works in this direction and this is one of the most actively developing area of research in holographic QCD. In particular, the situations with finite baryon density are very important, because lattice simulation is not efficient enough for such cases. In the following, we make a few comments on this issue.

Let us quickly outline what happens when we try to put baryons in high density. Recall that a baryon corresponds to a soliton in the five dimensional gauge theory 4.11. It can be shown that the size of the soliton is stabilized at a small value and it behaves like a small particle living in the five dimensional space-time. [22, 3] Due to the CS-term in 4.1], it is charged under the diagonal $U(1)$ part of the $U\left(N_{f}\right)$ gauge symmetry. Because of the non-trivial warp factors $h(z)$ and $k(z)$ in 4.1, the soliton has a potential in the $z$ direction and wants to sit near $z=0$.

If there are two baryons, they are repulsed by each other at short distances mainly because of the $U(1)$ charge. 228, 29, 30] The $U(1)$ part of the fluctuations of the 5 dim gauge field contains the component interpreted as the $\omega$-meson. The repulsive force is partly interpreted as that caused by the $\omega$-meson exchange. Next, consider an array of the baryons placed near $z=0$ along $x^{1 \sim 3}$ directions and increase the density. It was observed in [31 32, 33] that when the density is higher than a critical value, the baryons spread in the $z$ direction. (Figure 6) 


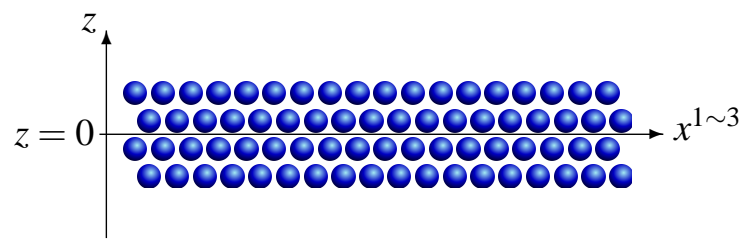

Figure 6: Baryons in high density.

This is an interesting phenomenon that involves the $z$ direction in an essential way. But, what is the interpretation of this phenomenon in QCD? The authors of [31 32 33] proposed to interpret it as formation of a fermi surface or a manifestation of quarkyonic phase. It would be interesting to investigate this issue further.

\section{Acknowledgments}

We would like to thank the organizers of the conference "Xth Quark Confinement and the Hadron Spectrum" for kind invitation and hospitality. We are also grateful to K. Hashimoto, H. Hata, T. Imoto, T. Sakai, and S. Yamato for pleasant collaborations.

\section{References}

[1] T. Sakai, S. Sugimoto, "Low energy hadron physics in holographic QCD,” Prog. Theor. Phys. 113, 843-882 (2005) [hep-th/0412141].

[2] T. Sakai, S. Sugimoto, "More on a holographic dual of QCD," Prog. Theor. Phys. 114, 1083-1118 (2005) [hep-th/0507073].

[3] H. Hata, T. Sakai, S. Sugimoto, S. Yamato, "Baryons from instantons in holographic QCD,” Prog. Theor. Phys. 117, 1157 (2007) [hep-th/0701280].

[4] K. Hashimoto, T. Sakai, S. Sugimoto, "Holographic Baryons: Static Properties and Form Factors from Gauge/String Duality,” Prog. Theor. Phys. 120, 1093-1137 (2008) [arXiv:0806.3122 [hep-th]].

[5] T. Imoto, T. Sakai, S. Sugimoto, “Mesons as Open Strings in a Holographic Dual of QCD,” Prog. Theor. Phys. 124, 263-284 (2010) [arXiv:1005.0655 [hep-th]].

[6] J. M. Maldacena, "The Large N limit of superconformal field theories and supergravity," Adv. Theor. Math. Phys. 2, 231 (1998) [hep-th/9711200].

[7] O. Aharony, S. S. Gubser, J. M. Maldacena, H. Ooguri and Y. Oz, "Large N field theories, string theory and gravity," Phys. Rept. 323, 183 (2000) [hep-th/9905111].

[8] E. Witten, "Anti-de Sitter space, thermal phase transition, and confinement in gauge theories," Adv. Theor. Math. Phys. 2, 505 (1998) [arXiv:hep-th/9803131].

[9] R. Casero, E. Kiritsis, A. Paredes: "Chiral symmetry breaking as open string tachyon condensation," Nucl. Phys. B 787, 98 (2007) [hep-th/0702155 [HEP-TH]]; O. Bergman, S. Seki, J. Sonnenschein: "Quark mass and condensate in HQCD,” JHEP 0712, 037 (2007) [arXiv:0708.2839 [hep-th]]; A. Dhar, P. Nag: "Sakai-Sugimoto model, Tachyon Condensation and Chiral symmetry Breaking," JHEP 0801, 055 (2008) [arXiv:0708.3233 [hep-th]]; "Tachyon condensation and quark mass in modified Sakai-Sugimoto model," Phys. Rev. D 78, 066021 (2008) [arXiv:0804.4807 [hep-th]]; 
O. Aharony, D. Kutasov: "Holographic Duals of Long Open Strings,” Phys. Rev. D 78, 026005 (2008) [arXiv:0803.3547 [hep-th]]; K. Hashimoto, T. Hirayama, F. -L. Lin, H. -U. Yee, "Quark Mass Deformation of Holographic Massless QCD,” JHEP 0807, 089 (2008) [arXiv:0803.4192 [hep-th]];

[10] K. Hashimoto, T. Hirayama and D. K. Hong, "Quark Mass Dependence of Hadron Spectrum in Holographic QCD,” Phys. Rev. D 81, 045016 (2010) [arXiv:0906.0402 [hep-th]]; K. Hashimoto, N. Iizuka, T. Ishii and D. Kadoh, "Three-flavor quark mass dependence of baryon spectra in holographic QCD,” Phys. Lett. B 691, 65 (2010) [arXiv:0910.1179 [hep-th]].

[11] E. Witten, "Baryons and branes in anti de Sitter space," JHEP 9807, 006 (1998) [hep-th/9805112].

[12] D. J. Gross and H. Ooguri, "Aspects of large N gauge theory dynamics as seen by string theory," Phys. Rev. D 58, 106002 (1998) [hep-th/9805129].

[13] D. T. Son, M. A. Stephanov, “QCD and dimensional deconstruction," Phys. Rev. D69, 065020 (2004) [hep-ph/0304182].

[14] M. Gell-Mann, D. Sharp and W. G. Wagner, "Decay Rates Of Neutral Mesons," Phys. Rev. Lett. 8, 261 (1962).

[15] T. Fujiwara, T. Kugo, H. Terao, S. Uehara and K. Yamawaki, "Nonabelian Anomaly And Vector Mesons As Dynamical Gauge Bosons Of Hidden Local Symmetries,” Prog. Theor. Phys. 73, 926 (1985).

[16] M. R. Douglas, "Branes within branes," In *Cargese 1997, Strings, branes and dualities* 267-275 [hep-th/9512077].

[17] T. H. R. Skyrme, "A Nonlinear field theory," Proc. Roy. Soc. Lond. A 260, 127 (1961); "Particle states of a quantized meson field," Proc. Roy. Soc. Lond. A 262, 237 (1961); "A Unified Field Theory Of Mesons And Baryons," Nucl. Phys. 31, 556 (1962).

[18] M. F. Atiyah and N. S. Manton, "SKYRMIONS FROM INSTANTONS," Phys. Lett. B 222, 438 (1989).

[19] G. S. Adkins, C. R. Nappi, E. Witten, "Static Properties of Nucleons in the Skyrme Model," Nucl. Phys. B228, 552 (1983).

[20] S. Sasaki, K. Sasaki, T. Hatsuda and M. Asakawa, "Bayesian approach to the first excited nucleon state in lattice QCD," Nucl. Phys. Proc. Suppl. 119, 302 (2003) [hep-lat/0209059].

[21] K. Sasaki, S. Sasaki and T. Hatsuda, "Spectral analysis of excited nucleons in lattice QCD with maximum entropy method," Phys. Lett. B 623, 208 (2005) [hep-lat/0504020].

[22] D. K. Hong, M. Rho, H. -U. Yee and P. Yi, "Chiral Dynamics of Baryons from String Theory," Phys. Rev. D 76, 061901 (2007) [hep-th/0701276 [HEP-TH]]; "Dynamics of baryons from string theory and vector dominance,” JHEP 0709, 063 (2007) [arXiv:0705.2632 [hep-th]].

[23] H. Hata, M. Murata and S. Yamato, "Chiral currents and static properties of nucleons in holographic QCD,” Phys. Rev. D 78, 086006 (2008) [arXiv:0803.0180 [hep-th]].

[24] K. -Y. Kim and I. Zahed, "Electromagnetic Baryon Form Factors from Holographic QCD," JHEP 0809, 007 (2008) [arXiv:0807.0033 [hep-th]].

[25] G. Panico and A. Wulzer, "Nucleon Form Factors from 5D Skyrmions," Nucl. Phys. A 825, 91 (2009) [arXiv:0811.2211 [hep-ph]].

[26] D. K. Hong, M. Rho, H. -U. Yee and P. Yi, "Nucleon form-factors and hidden symmetry in holographic QCD,” Phys. Rev. D 77, 014030 (2008) [arXiv:0710.4615 [hep-ph]]. 
[27] R. C. Brower, S. D. Mathur and C. -I Tan, "Glueball spectrum for QCD from AdS supergravity duality,” Nucl. Phys. B 587, 249 (2000) [hep-th/0003115].

[28] K. -Y. Kim and I. Zahed, "Nucleon-Nucleon Potential from Holography,” JHEP 0903, 131 (2009) [arXiv:0901.0012 [hep-th]].

[29] K. Hashimoto, T. Sakai and S. Sugimoto, "Nuclear Force from String Theory," Prog. Theor. Phys. 122, 427 (2009) [arXiv:0901.4449 [hep-th]].

[30] Y. Kim, S. Lee and P. Yi, “Holographic Deuteron and Nucleon-Nucleon Potential,” JHEP 0904, 086 (2009) [arXiv:0902.4048 [hep-th]].

[31] M. Rozali, H. -H. Shieh, M. Van Raamsdonk and J. Wu, "Cold Nuclear Matter In Holographic QCD," JHEP 0801, 053 (2008) [arXiv:0708.1322 [hep-th]].

[32] V. Kaplunovsky, D. Melnikov and J. Sonnenschein, “Baryonic Popcorn,” JHEP 1211, 047 (2012) [arXiv:1201.1331 [hep-th]].

[33] J. de Boer, B. D. Chowdhury, M. P. Heller and J. Jankowski, "Towards a holographic realization of the Quarkyonic phase," arXiv:1209.5915 [hep-th]. 